\title{
LINC01638 IncRNA mediates the postoperative distant recurrence of bladder cancer by upregulating ROCK2
}

\author{
SHOUXIAN YUAN ${ }^{1 *}$, XIUHUA LUAN $^{1 *}$, GUIQIANG HAN ${ }^{2}$, KECUN GUO $^{1}$, \\ SHENGHUI WANG $^{1}$ and XIANGKAI ZHANG ${ }^{3}$ \\ ${ }^{1}$ Department of Urology Surgery, The Second People's Hospital of Liaocheng; ${ }^{2}$ Department of Urology Surgery, \\ The People's Hospital of Liqing, Linqing, Shandong 252600; ${ }^{3}$ Department of Urology Surgery, \\ Shanxi Provincial People's Hospital, Taiyuan, Shanxi 030012, P.R. China
}

Received November 30, 2018; Accepted August 6, 2019

DOI: $10.3892 / 01.2019 .10924$

\begin{abstract}
It is well established that long intergenic non-protein coding RNA 1638 (LINC01638) promotes the development and progression of breast cancer, whereas its roles in other human diseases are currently unknown. In the present study, expression of LINC01638 and ROCK 2 was analyzed using quantitative PCR, ELISA and western blot. Receiver operating characteristic curve was used for diagnostic analysis. Cell transfections were performed to analyze interactions between LINC01638 and ROCK2, while Transwell assays were performed to analyze invasion and migration of the bladder cancer HT-1197 and HT-1376 cell lines. It was observed that LINC01638 and Rho-associated, coiled-coil containing protein kinase 2 (ROCK2) were significantly upregulated in the plasma of patients with early stage (stage I and II) bladder cancer compared with in healthy controls. Upregulation of LINC01638 and ROCK2 distinguished patients with early stage bladder cancer from healthy controls. Plasma levels of LINC01638 and ROCK2 were positively correlated in patients with bladder cancer, but not in healthy controls. A follow-up study after surgical resection revealed that LINC01638 and ROCK 2 were further upregulated in patients with distant recurrence, or distant and local recurrence, but not in patients with local recurrence and no recurrence. Overexpression of LINC01638 led to ROCK2 upregulation in bladder cancer cells, whereas ROCK2 overexpression did not significantly affect LINC01638 expression. Overexpression of LINC01638 and ROCK2 mediated the promoted migration and invasion
\end{abstract}

Correspondence to: Dr Shenghui Wang, Department of Urology Surgery, The Second People's Hospital of Liaocheng, 306 Jiankang Street, Linqing, Shandong 252600, P.R. China

E-mail: vr56247@163.com

${ }^{*}$ Contributed equally

Key words: bladder cancer, long intergenic non-protein coding RNA 1638, Rho-associated, coiled-coil containing protein kinase 2, recurrence of bladder cancer cells, and ROCK2 small interfering RNA silencing attenuated the enhancing effects of LINC01638 on cancer cell migration and invasion. Therefore, LINC01638 may mediate the postoperative distant recurrence of bladder cancer by upregulating ROCK2.

\section{Introduction}

Bladder cancer is the ninth most prevalent type of cancer worldwide (1). In 2012, there were 430,000 new cases of bladder cancer and 165,000 deaths as a result of the disease worldwide (2), with $>60 \%$ of new bladder cancer cases and approximately one half of deaths occurring in less developed regions (2). The clinical treatment of bladder cancer is challenged by tumor metastasis in patients with advanced stages of the disease (3). Although surgical resection usually results in satisfactory outcomes in patients with early stage bladder cancer (4), high postoperative recurrence rates lead to unacceptably high mortality rates (5). Therefore, preventing recurrence following treatment is critical for the survival of patients with bladder cancer.

Rho-associated protein kinase 2 (ROCK2) is a member of the protein kinase $\mathrm{A} / \mathrm{G} / \mathrm{C}$ family of serine-threonine kinases involved in cell movement by regulating the cytoskeleton (6). Due to the regulatory roles in cellular behavior, ROCK 2 has been demonstrated to be a key player in human cancer (7), and the inhibition of ROCK2 is considered a promising therapeutic mechanism for cancer treatment (8). ROCK2 participates in the development of cancer through interactions with multiple factors, including long non-coding RNAs (lncRNAs) (9), which are a group of non-protein coding RNAs involved in almost all critical aspects of cancer biology (10). Long intergenic non-protein coding RNA 1638 (LINC01638) promotes breast cancer (11), while its roles in other human diseases are currently unknown. The preliminary RNA-sequencing data of the present study revealed the overexpression of LINC01638 in bladder cancer tissues and its inverse correlation with ROCK2 mRNA (data not shown), indicating the potential role of LINC01638 in bladder cancer. The aim of the present study was to investigate the potential interaction between LINC01638 and ROCK1 in bladder cancer. 


\section{Materials and methods}

Patients and cell lines. A total of 88 patients with bladder cancer from The Second People's Hospital of Liaocheng (Linqing, China), The People's Hospital of Liqing (Linqing, China) and Shanxi Provincial People's Hospital Urology Surgery (Taiyuan, China) between March 2013 and March 2015 were analyzed in the present study. The inclusion criteria were as follows: i) Bladder cancer confirmed by pathological biopsy; ii) stage I or II bladder cancer; iii) patients appropriate for surgical resection (based on their health conditions and clinical stages); and iv) patients who had complete 3-year follow-up data. The exclusion criteria were as follows: i) Patients diagnosed with multiple diseases; ii) patients that were treated prior to admission; and iii) patients that failed to complete the follow-up or died before the diagnosis of recurrence. The 88 patients included 60 males and 28 females, and age ranged from 36-67 years, with a mean age of $45.4 \pm 4.3$ (standard deviation) years. In addition, 68 healthy volunteers that had received systemic physiological examinations in The Second People's Hospital of Liaocheng, The People's Hospital of Liqing or Shanxi Provincial People's Hospital Urology Surgery during the same time period were included to serve as the control (C) group. The $\mathrm{C}$ group included 47 males and 21 females, and age ranged from 33-69 years, with a mean age of 46.2 \pm 5.1 (standard deviation) years. The patient and $\mathrm{C}$ groups all had similar age and sex distributions (revealed using $\chi^{2}$ analysis). This study was approved by the Ethics Committee of The Second People's Hospital of Liaocheng, The People's Hospital of Liqing and Shanxi Provincial People's Hospital Urology Surgery. All patients included in the study provided written informed consent.

The two human bladder cancer cell lines HT-1197 and HT-1376 (American Type Culture Collection; ATCC) were used in the present study to perform all in vitro cell experiments. Cells were cultivated in Eagle's minimum essential medium containing $10 \% \mathrm{FBS}$ (both ATCC) at $37^{\circ} \mathrm{C}$ in a humidified incubator with $5 \% \mathrm{CO}_{2}$.

Follow-up and specimens. Fasting blood $(5 \mathrm{ml})$ was extracted from the elbow vein of each participant 1 day after admission (before treatment). All patients with bladder cancer received surgical resection and were followed up for 3 years after discharge. Fasting blood $(5 \mathrm{ml})$ was extracted from patients on the day of discharge, or on the day of the diagnosis of recurrence or at the end of follow-up in cases of non-recurrence (NR). Plasma samples were prepared by centrifuging the blood samples in EDTA tubes at room temperature for $10 \mathrm{~min}$ at $1,200 \mathrm{xg}$.

Reverse transcription-quantitative PCR (RT-qPCR). To detect the expression of LINC01638 and ROCK2 mRNA, total RNA was extracted from plasma, and HT-1197 and HT-1376 cells using TRIzol ${ }^{\circledR}$ reagent (Thermo Fisher Scientific, Inc.), RT was performed using SuperScript III Reverse Transcriptase kit (Thermo Fisher Scientific, Inc.) at the following conditions: $50^{\circ} \mathrm{C}$ for $20 \mathrm{~min}$ and $80^{\circ} \mathrm{C}$ for $10 \mathrm{~min}$. qPCR were prepared using SYBR ${ }^{\circledR}$ Green PCR Master Mix (Thermo Fisher Scientific, Inc.) and the following thermocycling conditions were used: Initial denaturation at $95^{\circ} \mathrm{C}$ for $1 \mathrm{~min} ; 40$ cylcles of $95^{\circ} \mathrm{C}$ for $10 \mathrm{sec}$ and $58^{\circ} \mathrm{C}$ for $40 \mathrm{sec}$. Primers for LINC01638, ROCK 2 and endogenous control GAPDH were synthesized by Invitrogen (Thermo Fisher Scientific, Inc.). The following primer sequences were used: ROCK2 forward, 5'-CTAGGC CGGGCGAAG-3 and reverse 5'-TCCAGCTTCCTCTGACGA C-3'; LINC01638 forward, 5'-AATACATCAGCACTGTTG CCTTT-3' and reverse 5'-CTCCATACATACATCTCCAAA AAGT-3'; GAPDH forward, 5'-AAGGTGAAGGTCGGAGTC AA-3' and reverse 5'-AATGAAGGGGTCATTGATGG-3'. The $2^{-\Delta \Delta \mathrm{Cq}}$ method (12) was used to normalize the expression of LINC01638 and ROCK2 to endogenous control GAPDH.

Vectors and cell transfection. Vectors expressing LINC01638 or ROCK2 and empty vectors were constructed by Sangon Biotech Co., Ltd using the pcDNA3.1 vector as the primary vector. ROCK2 small interfering (si)RNA (human; cat. no. sc-29474) and negative control (NC) siRNA (cat. no. sc-37007) were purchased from Santa Cruz Biotechnology, Inc. Lipofectamine ${ }^{\circledR} 2000$ reagent (Invitrogen; Thermo Fisher Scientific, Inc.) was used to perform all cell transfections with $10 \mathrm{nM}$ vectors or $50 \mathrm{nM}$ siRNA and using aforementioned cell lines at a density of $1 \times 10^{6}$ cells in $2 \mathrm{ml}$ cell suspension. Treatment with Lipofectamine ${ }^{\circledR} 2000$ reagent alone was used as the $\mathrm{C}$ group and transfection with empty vectors or NC siRNA was used as the NC group. Cells were used for subsequent experiments when LINC01638 and ROCK 2 overexpression rates were $>200 \%$ and ROCK2 knockdown rate was $<50 \%$, only. The time interval between transfection and subsequent experimentation was $24 \mathrm{~h}$.

In vitro migration and invasion assay. Following transfection, cell suspensions were prepared and cell density was adjusted to $5 \times 10^{4}$ cells $/ \mathrm{ml}$ using serum-free cell culture medium (as aforementioned). Cell suspensions were transferred to the upper chamber at $0.1 \mathrm{ml}$ per well, while the lower chamber was filled with cell culture medium containing $20 \%$ FBS (Sigma-Aldrich; Merck KGaA). Cells were cultured for $2 \mathrm{~h}$, followed by staining of the upper chamber membrane with $0.5 \%$ crystal violet (Sigma-Aldrich; Merck KGaA) for $20 \mathrm{~min}$ at room temperature. Cell migration and invasion assays were performed in the same way except that the upper chamber was pre-coated with Matrigel (cat. no. 356234; Corning Incorporated) prior to the invasion assay. Invading and migrating cells were observed under a light microscope (x40 magnification) and 5 visual fields were selected to count cells.

Western blot analysis. To detect the expression levels of the ROCK 2 protein, total protein was extracted using RIPA solution (Thermo Fisher Scientific, Inc.), protein concentration was determined by bicinchoninic acid assay and all protein samples were separated via SDS-PAGE (10\% gel) electrophoresis (30 $\mu \mathrm{g}$ per well) following denaturation. Following this, the proteins were transferred to PVDF membranes, which were blocked in 5\% skimmed milk in PBS membrane at room temperature for $2 \mathrm{~h}$. Subsequently, membranes were further incubated with primary antibodies against rabbit anti-human ROCK2 (1:1,200; cat. no. ab71598; Abcam) and anti-GAPDH (1:1,200; cat. no. ab9485; Abcam) for $12 \mathrm{~h}$ at $4^{\circ} \mathrm{C}$, followed by incubation with $\mathrm{IgG}$-horseradish peroxidase secondary 

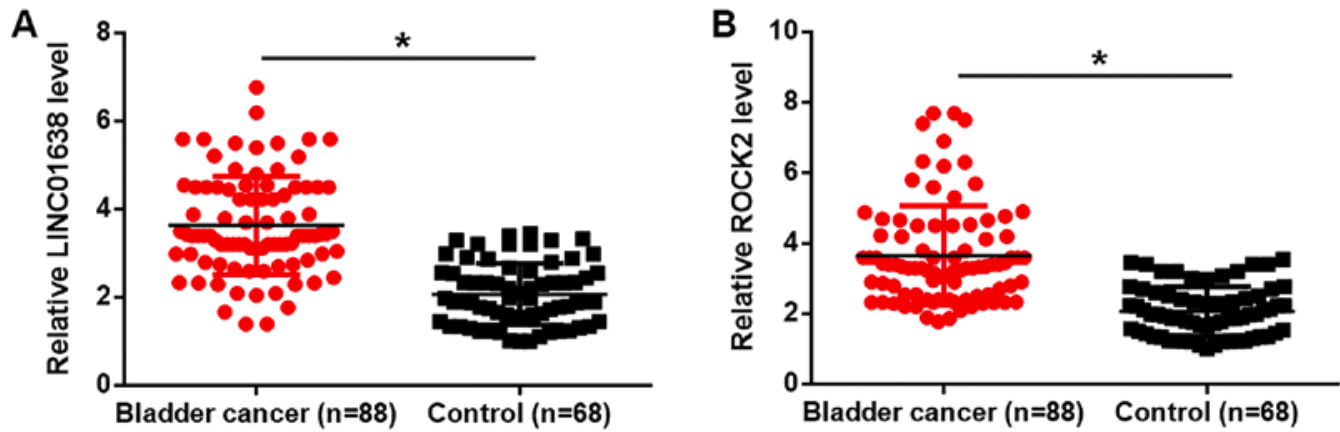

Figure 1. Plasma LINC01638 and ROCK2 mRNA is upregulated in patients with bladder cancer. Reverse transcription-quantitative PCR was performed to investigate the differential expression of LINC01638 and ROCK2 in patients with bladder cancer and healthy controls. The results revealed that plasma (A) LINC01638 and (B) ROCK2 mRNA were significantly upregulated in patients with bladder cancer compared with healthy controls. "P<0.05. LINC01638, long intergenic non-protein coding RNA 1638; ROCK2, Rho-associated, coiled-coil containing protein kinase 2.
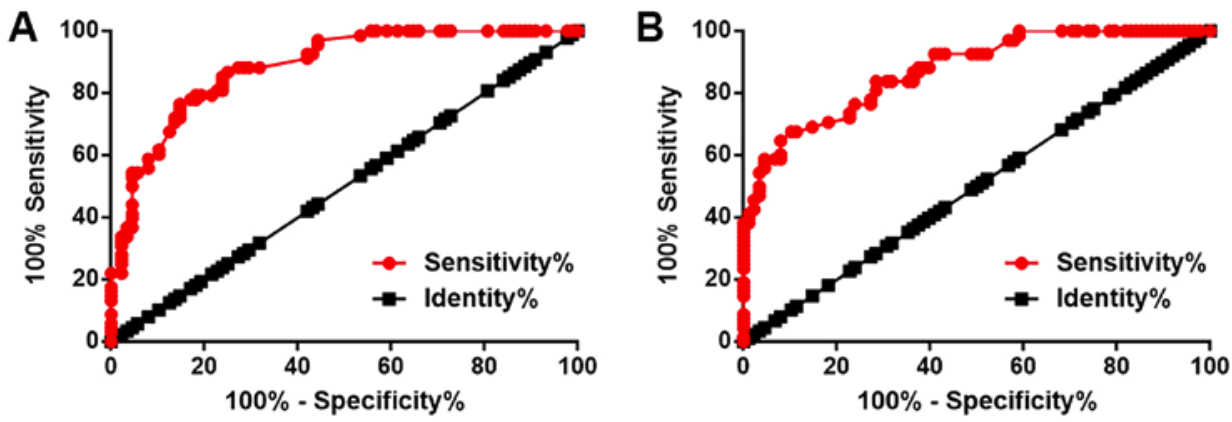

Figure 2. Altered plasma levels of LINC01638 and ROCK2 mRNA distinguish patients with bladder cancer from healthy controls. Receiver operating characteristic curve analysis was performed to investigate the diagnostic potentials of LINC01638 and ROCK2 in bladder cancer. It was observed that the altered plasma levels of (A) LINC01638 and (B) ROCK2 mRNA distinguish patients with bladder cancer from healthy controls. LINC01638, long intergenic non-protein coding RNA 1638; ROCK2, Rho-associated, coiled-coil containing protein kinase 2.

antibody (1:1,200; cat. no. MBS435036; MyBioSource, Inc.) for $2 \mathrm{~h}$ at room temperature. Signals were developed using ECL (Sigma-Aldrich; Merck KGaA) and analyzed using ImageJ software v1.46 (National Institutes of Health).

Statistical analysis. All experiments were performed in 3 independent biological replicates and data are expressed as the mean \pm standard deviation. Comparisons between patients and controls were performed using an unpaired t-test. Comparisons between the pre-treatment and follow-up data within the same group were performed using a paired t-test. Comparisons among multiple groups were performed using one-way ANOVA and Tukey's test. Receiver operating characteristic (ROC) curve analysis was performed to evaluate the diagnostic value of plasma LINC01638 and ROCK 2 mRNA for bladder cancer. Correlation between the expression levels of LINC01638 and ROCK2 were performed using Pearson's correlation coefficient. $\mathrm{P}<0.05$ was considered to indicate a statistically significant difference.

\section{Results}

Plasma LINC01638 and ROCK2 mRNA is upregulated in patients with bladder cancer. RT-qPCR was performed to detect the plasma LINC01638 and ROCK2 mRNA in patients with bladder cancer and healthy controls. As presented in
Fig. 1, compared with healthy controls, plasma LINC01638 (Fig. 1A) and ROCK2 mRNA (Fig. 1B) were significantly higher in patients with bladder cancer $(\mathrm{P}<0.05)$. This data suggests that overexpression of LINC01638 and ROCK2 may have a role in bladder cancer.

Altered plasma levels of LINC01638 and ROCK2 $\mathrm{mRNA}$ distinguish patients with bladder cancer from healthy controls. A ROC curve analysis was performed to evaluate the diagnostic value of plasma LINC01638 and ROCK2 mRNA for bladder cancer, with bladder cancer as true positive cases and healthy controls as true negative cases. For plasma LINC01638, the area under the curve was 0.88 , with a standard error of 0.026 and $95 \%$ confidence interval of 0.83-0.94 (Fig. 2A). For plasma ROCK2, area under the curve was 0.87 , with a standard error of 0.027 and $95 \%$ confidence interval of 0.82-0.93 (Fig. 2B). Therefore, plasma LINC01638 and ROCK2 mRNA may serve as diagnostic biomarkers for bladder cancer.

Plasma levels of LINC01638 and ROCK2 are positively correlated in bladder cancer patients but not in healthy controls. Correlations between the expression levels of LINC01638 and ROCK2 were performed by Pearson's correlation coefficient. As presented in Fig. 3A, the plasma levels of LINC01638 and ROCK2 were positively and significantly correlated in patients with bladder cancer (Fig. 3A). However, 

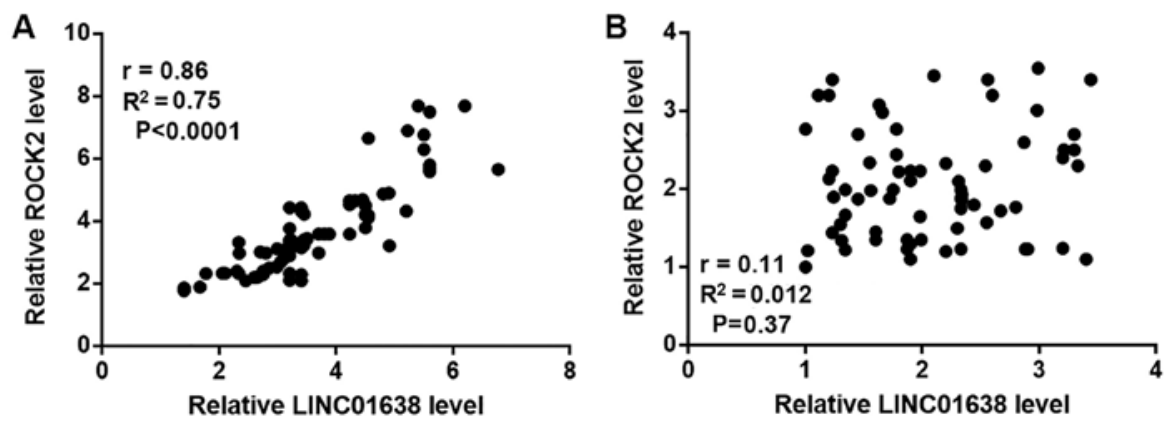

Figure 3. Plasma levels of LINC01638 and ROCK2 are positively correlated in patients with bladder cancer but not in healthy controls. Pearson's correlation coefficient was used to analyze the correlation between plasma levels of LINC01638 and ROCK2. (A) Plasma levels of LINC01638 and ROCK2 were significantly and positively correlated in patients with bladder cancer. (B) Plasma levels of LINC01638 and ROCK2 were not significantly correlated in healthy controls. LINC01638, long intergenic non-protein coding RNA 1638; ROCK2, Rho-associated, coiled-coil containing protein kinase 2.

A

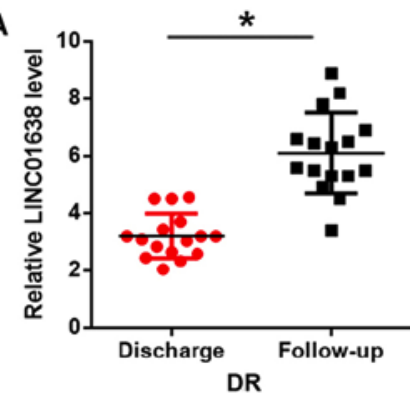

B

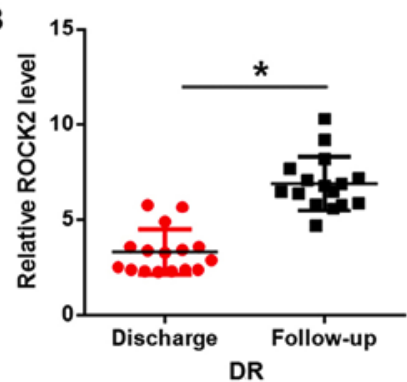

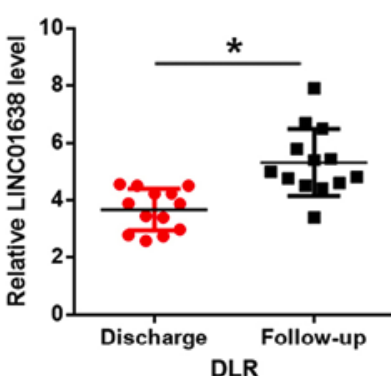
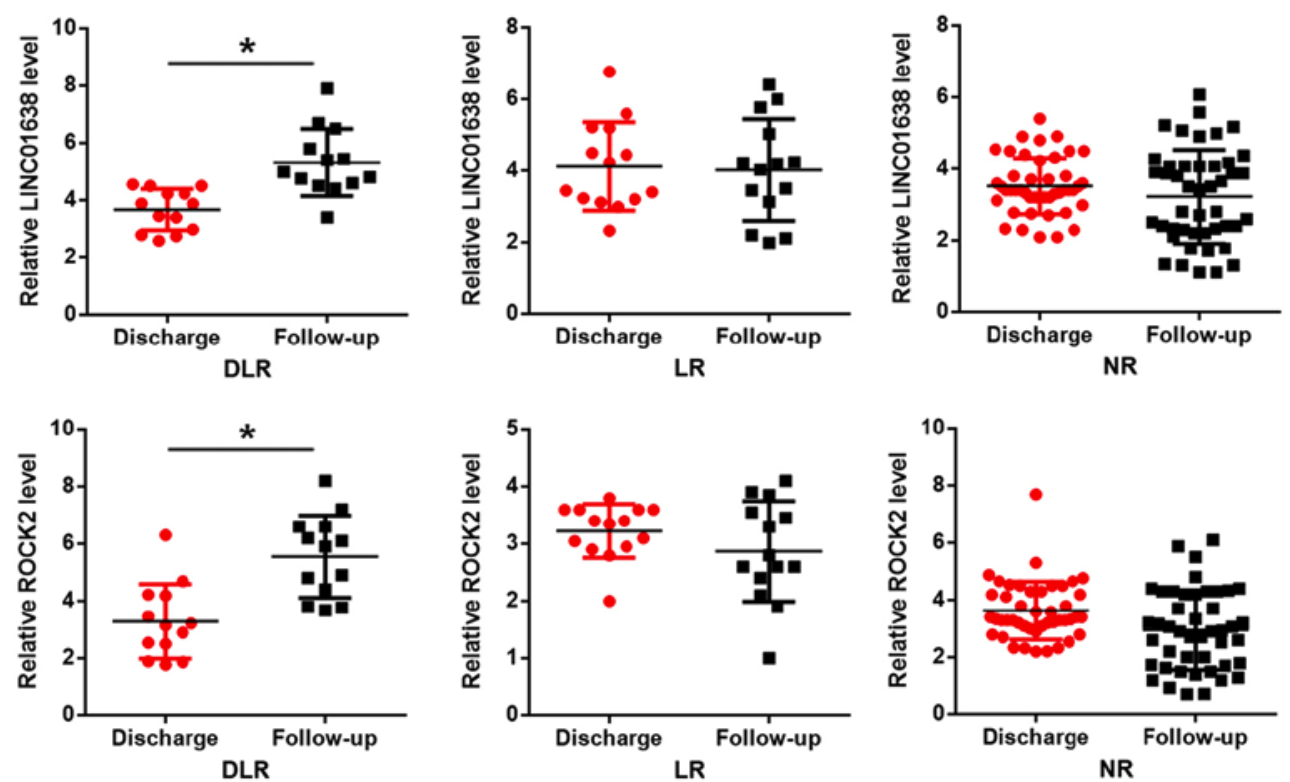

Figure 4. LINC01638 and ROCK2 upregulation is induced by DR. Reverse transcription-quantitative PCR was performed to detect the expression levels of LINC01638 and ROCK2 in patients with bladder cancer that had different recurrence conditions. The results revealed that, compared with discharge level, plasma levels of (A) LINC01638 and (B) ROCK2 were significantly increased in the DR and DLR groups, but not in LR and NR groups. "P<0.05. LINC01638, long intergenic non-protein coding RNA 1638; ROCK2, Rho-associated, coiled-coil containing protein kinase 2; DR, distant recurrence; DLR, distant and local recurrence; LR, local recurrence; NR, non-recurrence.

no significant correlation was observed between the plasma levels of LINC01638 and ROCK2 in healthy controls (Fig. 3B). Therefore, there may be interaction between LINC01638 and ROCK2 in bladder cancer, but not under normal physiological conditions.

LINC01638 and ROCK2 upregulation is induced by distant recurrence $(D R)$. Based on the follow-up data, there were 16 cases of DR, 12 cases of both distant and local recurrence (DLR), 14 cases of local recurrence (LR) only and 46 cases of NR. Compared with the level at discharge, the plasma levels of LINC01638 (Fig. 4A) and ROCK2 (Fig. 4B) were increased in the DR and DLR groups $(\mathrm{P}<0.05)$, but not in LR and NR groups (day of the diagnosis of recurrence or at the end of follow-up in cases of non-recurrence). Therefore, LINC01638 and ROCK2 may specifically participate in the DR of bladder cancer.
Overexpression of LINC01638 leads to ROCK2 upregulation in bladder cancer cells. Compared with the C and NC, overexpression of LINC01638 led to ROCK2 upregulation in the bladder cancer cell lines HT-1197 and HT-1376 at the mRNA ( $\mathrm{P}<0.05$; Fig. 5A) and protein levels ( $\mathrm{P}<0.05$; Fig. 5B). However, ROCK2 overexpression failed to significantly affect the expression of LINC01638 (Fig. 5C). These data suggest that LINC01638 may serve as an upstream positive regulator of ROCK2 in bladder cancer.

Overexpression of LINC01638 promotes bladder cancer cell migration and invasion through ROCK2. Successful knockdown of ROCK2 was established in the two cell lines ( $\mathrm{P}<0.05$; Fig. 6). Compared with $\mathrm{C}$ and $\mathrm{NC}$, overexpression of LINC01638 and ROCK2 promoted the migration (Fig. 7A) and invasion (Fig. 7B) of bladder cancer cell lines HT-1197 and HT-1376 $(\mathrm{P}<0.05)$, while ROCK2 siRNA silencing attenuated 

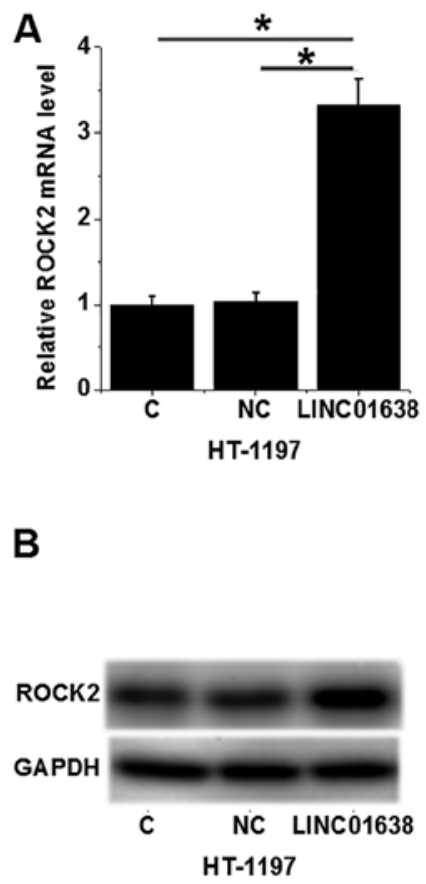
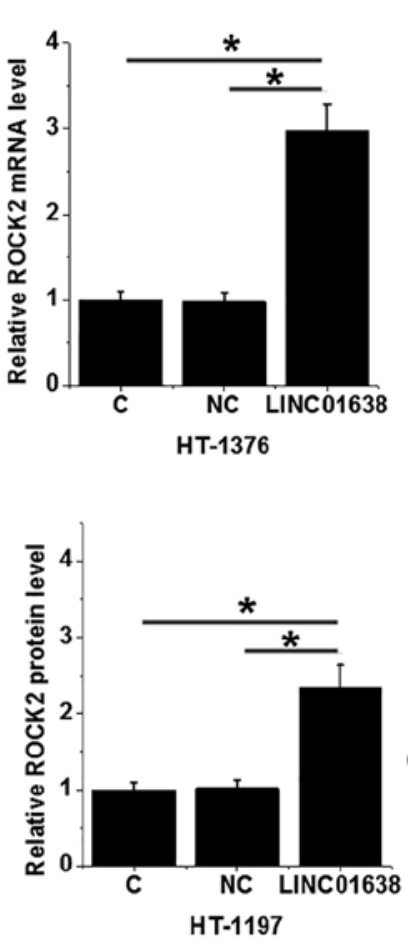
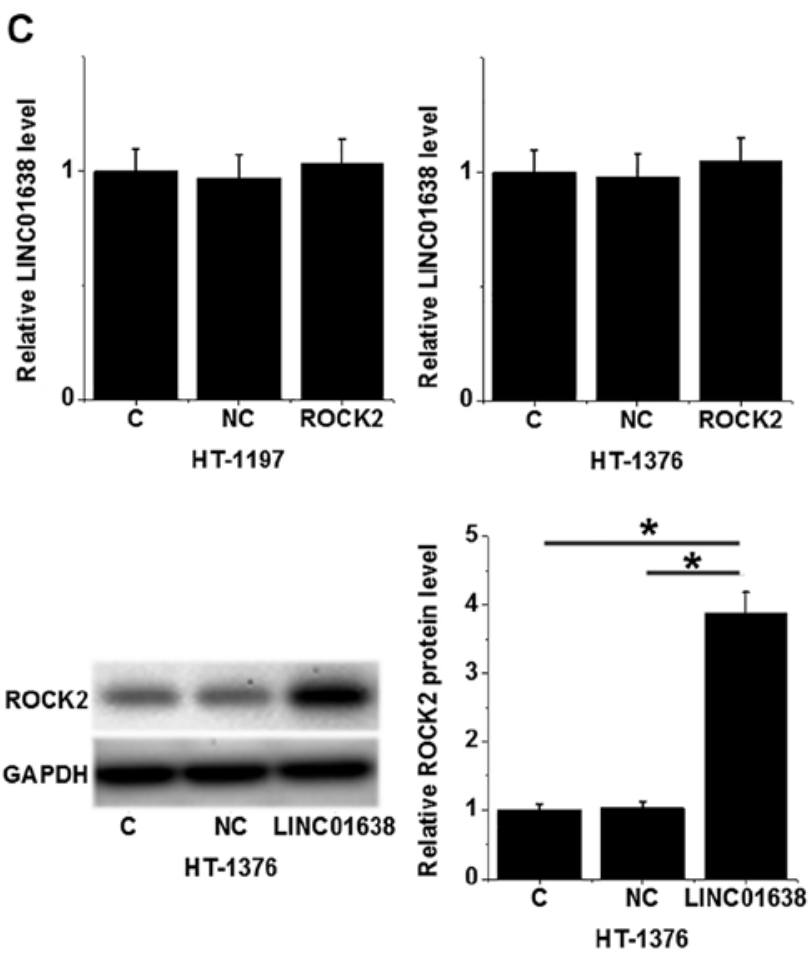

Figure 5. Overexpression of LINC01638 leads to ROCK2 upregulation in bladder cancer cells. Overexpression of LINC01638 and ROCK2 and reverse transcription-quantitative PCR experiments were performed to investigate the interaction between LINC01638 and ROCK2 in bladder cancer. Overexpression of LINC01638 led to ROCK2 upregulation in cells of bladder cancer cell lines HT-1197 and HT-1376 at both (A) mRNA and (B) protein levels. (C) ROCK2 overexpression failed to significantly affect the expression of LINC01638. ${ }^{*} \mathrm{P}<0.05$. LINC01638, long intergenic non-protein coding RNA 1638 ; ROCK2, Rho-associated, coiled-coil containing protein kinase 2; $\mathrm{C}$, control; $\mathrm{NC}$, negative control.
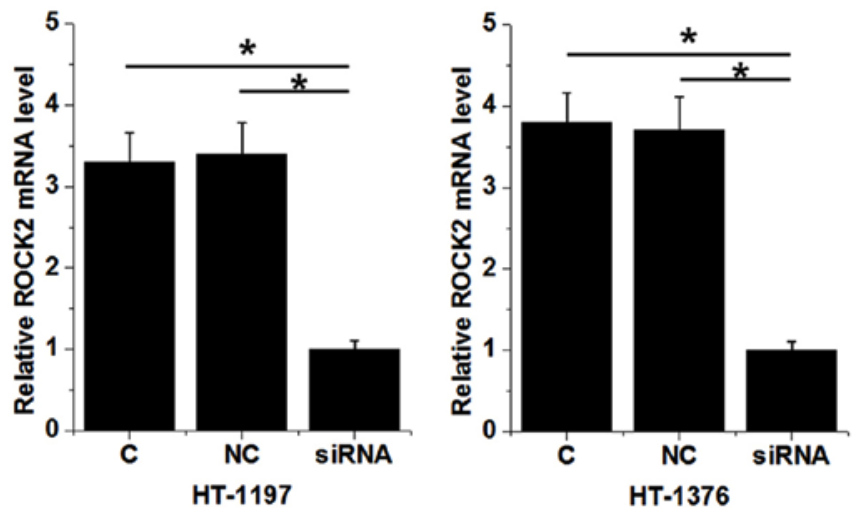

Figure 6. Knockdown of ROCK2 in bladder cancer cell lines HT-1197 and HT-1376 following siRNA transfection. ${ }^{*} \mathrm{P}<0.05$. ROCK2, Rho-associated, coiled-coil containing protein kinase 2 ; $\mathrm{C}$, control; $\mathrm{NC}$, negative control; siRNA, small interfering RNA.

the effect of LINC01638 on cancer cell migration and invasion. These data indicate that LINC01638 may promote the migration and invasion of bladder cancer cells via upregulation of ROCK2.

\section{Discussion}

The high recurrence rate is a major challenge in the treatment of bladder cancer. The main result of the present study is that LINC01638 appears to be involved in the DR of bladder cancer. The actions of LINC01638 are likely mediated by ROCK 2 .
ROCK2 mediates cancer cell migration and invasion (13), with downregulation of ROCK2 inhibiting tumor metastasis $(14,15)$. To the best of our knowledge, studies investigating the involvement of ROCK 2 in bladder cancer are rare. The present study, demonstrated the upregulated expression of ROCK2 in patients with bladder cancer and the effect of ROCK 2 on bladder cancer cell migration and invasion. These results indicate that ROCK2 may have an oncogenic role in bladder cancer.

The present study demonstrated that LINC01638 may be an oncogenic IncRNA in bladder cancer as LINC01638 and ROCK2 mRNA were detected in plasma of patients with bladder cancer, which indicated that LINC01638 and ROCK 2 mRNA can be released into the blood after synthesis, possibly from multiple organs but further investigation is required. In addition, it was indicated that LINC01638 is likely an upstream activator of ROCK2 in the regulation of bladder cancer migration and invasion. Overexpression of LINC01638 led to the upregulation of ROCK2 at the mRNA and protein levels. It is known that lncRNAs can participate in cancer biology by regulating oncogene or tumor suppressor expression at the post-transcriptional and translational levels, such as recruiting a regulatory protein complex or inhibiting the binding of a transcriptional regulatory proteins, such as transcription factors (10). Therefore, LINC01638 may affect ROCK 2 mRNA degradation and possibly also ROCK2 translation, which in turn regulates the ROCK2 protein level. The upregulated expression of LINC01638 and ROCK2 in patients with DR suggest that LINC01638 may promote postoperative DR of bladder cancer by promoting the 
A
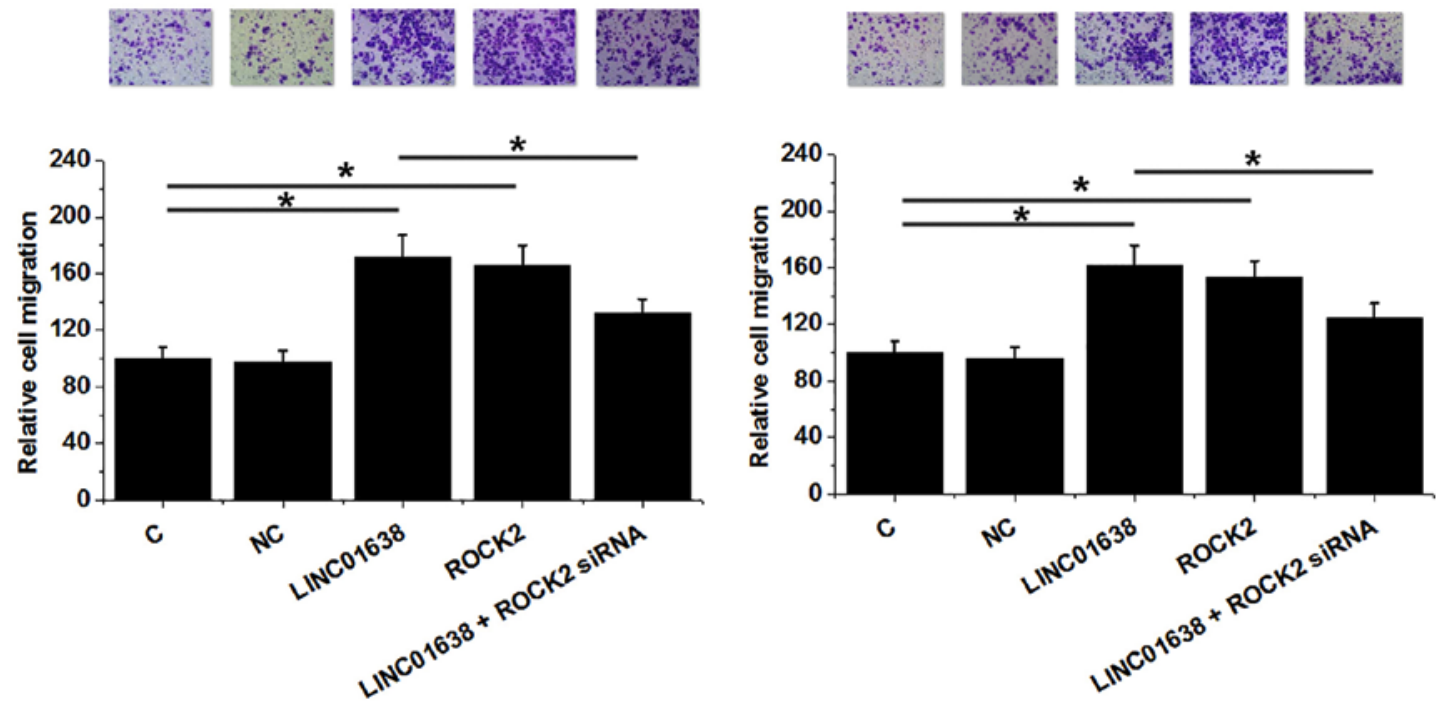

B

HT-1197

HT-1376
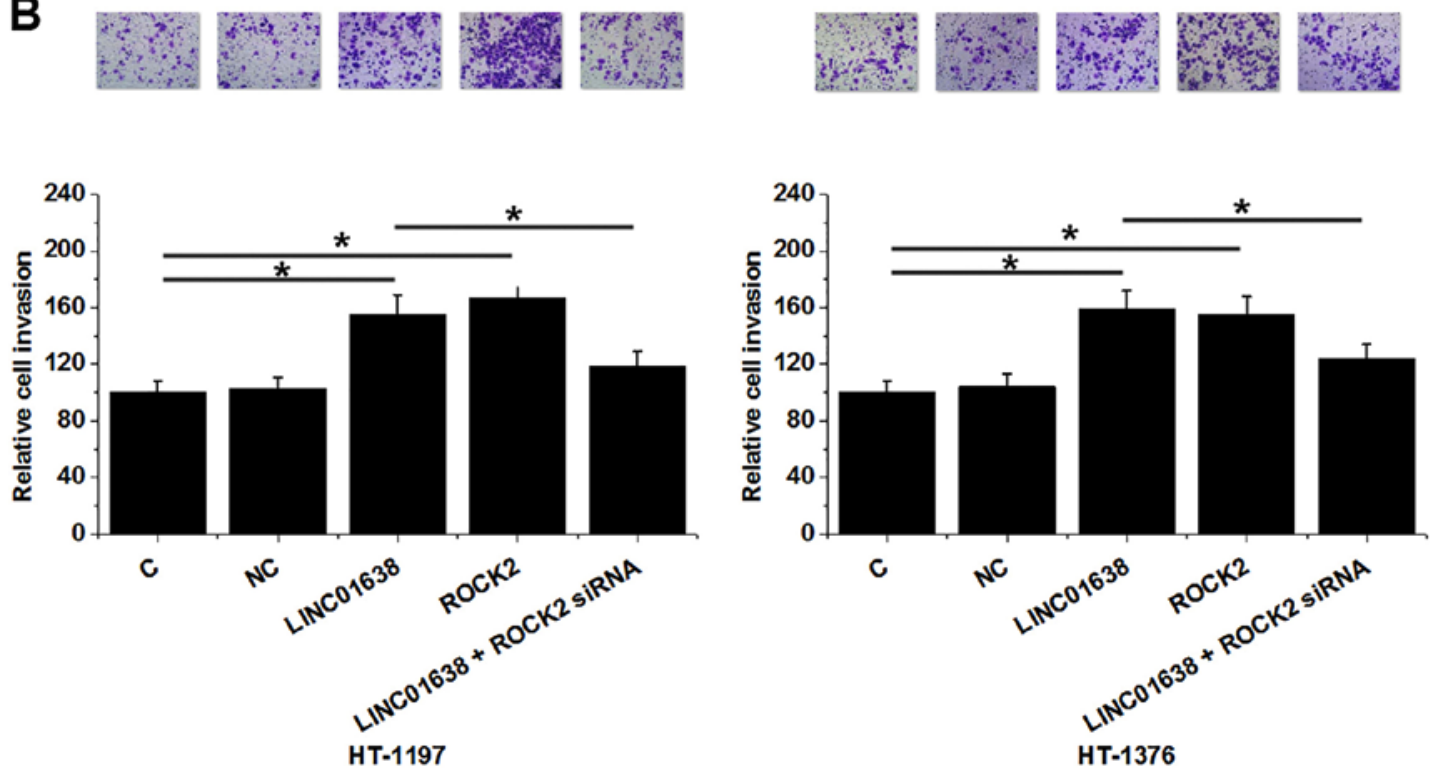

Figure 7. Overexpression of LINC01638 promotes bladder cancer cell migration and invasion through ROCK2. Transwell migration and invasion assays were performed to investigate the effects of LINC01638 and ROCK2 in bladder cancer cell migration and invasion. Overexpression of LINC01638 and ROCK2 mediated the promoted (A) migration and (B) invasion of cells of bladder cancer cell lines HT-1197 and HT-1376, whereas ROCK2 siRNA silencing attenuated the enhancing effects of LINC01638 on cancer cell migration and invasion. ${ }^{*} \mathrm{P}<0.05$. NC and empty vector were used as controls for overexpression. LINC01638, long intergenic non-protein coding RNA 1638; ROCK2, Rho-associated, coiled-coil containing protein kinase 2; siRNA, small interfering RNA; $\mathrm{C}$, control; $\mathrm{NC}$, negative control.

expression of ROCK2. However, further in vivo experiments, such as animal models, are required in order to confirm this conclusion.

The low diagnosis rate of bladder cancer in the early stages limits the used of surgical resection, which is currently the only radical treatment for solid bladder tumors (15). Despite the efforts made to develop of novel diagnostic approaches, such as biomarkers (16) and imaging techniques (17), the rate of early bladder cancer diagnosis remains low. In the present study, it was demonstrated that high levels of circulating LINC01638 and ROCK2 in the plasma may be used to effectively distinguish patients with early stage bladder cancer from healthy controls, indicating the potential of LINC01638 and ROCK2 for use in the early diagnosis of bladder cancer. However, these two factors should be combined with other markers in order to increase the diagnostic specificity.

The present study only included two cancer cell lines. Future studies should include additional cancer cell lines in order to perform in-depth analysis of the correlation between LINC01638 expression and cancer cell behavior.

It is worth noting that LINC01638 is a novel lncRNA, with its functions currently only established in breast cancer $(11,18)$. In breast cancer, LINC01638 inhibits speckle-type POZ protein-mediated c-Myc degradation to activate metadherin-Twist1 signaling (11). Similar interactions may exist in bladder cancer, and future studies will explore this. 
In conclusion, LINC01638 and ROCK2 have potential oncogenic roles in bladder cancer. LINC01638 lncRNA may be associated with postoperative DR of bladder cancer by upregulating ROCK2.

\section{Acknowledgements}

Not applicable.

\section{Funding}

No funding was received.

\section{Availability of data and materials}

The datasets used and/or analyzed during the current study are available from the corresponding author on reasonable request.

\section{Authors' contributions}

SY, XL and SW designed the experiments. SY, XL and GH performed the experiments. KG and XZ collected and analyzed the data. SW drafted the manuscript. All authors approved the manuscript for publication.

\section{Ethics approval and consent to participate}

This study was approved by the Ethics Committee of The Second People's Hospital of Liaocheng, The People's Hospital of Liqing and Shanxi Provincial People's Hospital Urology Surgery. All patients included in the study provided written informed consent.

\section{Patient consent for publication}

Not applicable.

\section{Competing interests}

The authors declare that they have no competing interests.

\section{References}

1. Antoni S, Ferlay J, Soerjomataram I, Znaor A, Jemal A and Bray F: Bladder cancer incidence and mortality: A global overview and recent trends. Eur Urol 71: 96-108, 2017.

2. Torre LA, Siegel RL, Ward EM and Jemal A: Global cancer incidence and mortality rates and trends-an update. Cancer Epidemiol Biomarkers Prev 25: 16-27, 2016.

3. Witjes JA, Compérat E, Cowan NC, De Santis M, Gakis G, Lebret T, Ribal MJ, Van der Heijden AG and Sherif A; European Association of Urology: EAU guidelines on muscle-invasive and metastatic bladder cancer: Summary of the 2013 guidelines. Eur Urol 65: 778-792, 2014.
4. Naito S, Algaba F, Babjuk M, Bryan RT, Sun YH, Valiquette L and de la Rosette J; CROES Narrow Band Imaging Global Study Group: The clinical research office of the endourological society (CROES) multicentre randomised trial of narrow band imaging-assisted transurethral resection of bladder tumour (TURBT) versus conventional white light imaging-assisted TURBT in primary non-muscle-invasive bladder cancer patients: Trial protocol and 1-year results. Eur Urol 70: 506-515, 2016.

5. Knowles MA and Hurst CD: Molecular biology of bladder cancer: New insights into pathogenesis and clinical diversity. Nat Rev Cancer 15: 25-41, 2015.

6. Riento K and Ridley AJ: Rocks: Multifunctional kinases in cell behaviour. Nat Rev Mol Cell Biol 4: 446-456, 2003.

7. Wei L, Surma M, Shi S, Lambert-Cheatham N and Shi J: Novel insights into the roles of Rho kinase in cancer. Arch Immunol Ther Exp (Warsz) 64: 259-278, 2016.

8. Rath $\mathrm{N}$ and Olson MF: Rho-associated kinases in tumorigenesis: Re-considering ROCK inhibition for cancer therapy. EMBO Rep 13: 900-908, 2012.

9. Tang Y, He Y, Zhang P, Wang J, Fan C, Yang L, Xiong F, Zhang S, Gong Z, Nie S, et al: lncRNAs regulate the cytoskeleton and related Rho/ROCK signaling in cancer metastasis. Mol Cancer 17: 77, 2018.

10. Yang G, Lu X and Yuan L: lncRNA: A link between RNA and cancer. Biochim Biophys Acta 1839: 1097-1109, 2014.

11. Luo L, Tang H, Ling L, Li N, Jia X, Zhang Z, Wang X, Shi L, Yin J, Qiu N, et al: LINC01638 lncRNA activates MTDH-Twist1 signaling by preventing SPOP-mediated c-Myc degradation in triple-negative breast cancer. Oncogene 37: 6166-6179, 2018.

12. Livak KJ and Schmittgen TD: Analysis of relative gene expression data using real-time quantitative PCR and the 2(-Delta Delta C(T)) method. Methods 25: 402-408, 2001.

13. Wang W, Zhou X and Wei M: MicroRNA-144 suppresses osteosarcoma growth and metastasis by targeting ROCK1 and ROCK2. Oncotarget 6: 10297-10308, 2015.

14. Kroiss A, Vincent S, Decaussin-Petrucci M, Meugnier E, Viallet J, Ruffion A, Chalmel F, Samarut J and Allioli N: Androgen-regulated microRNA-135a decreases prostate cancer cell migration and invasion through downregulating ROCK1 and ROCK2. Oncogene 34: 2846-2855, 2015.

15. Bellmunt J, Orsola A, Leow JJ, Wiegel T, De Santis M and Horwich A; ESMO Guidelines Working Group: Bladder cancer: ESMO practice guidelines for diagnosis, treatment and follow-up. Ann Oncol 25 (Suppl 3): iii40-iii48, 2014.

16. Schmitz-Dräger BJ, Droller M, Lokeshwar VB, Lotan Y, Hudson MA, van Rhijn BW, Marberger MJ, Fradet Y, Hemstreet GP, Malmstrom PU, et al: Molecular markers for bladder cancer screening, early diagnosis, and surveillance: The WHO/ICUD consensus. Urol Int 94: 1-24, 2015.

17. Zheng C, Lv Y, Zhong Q, Wang R and Jiang Q: Narrow band imaging diagnosis of bladder cancer: Systematic review and meta-analysis. BJU Int 110: E680-E687, 2012.

18. Liu P, Tang H, Wu J, Qiu X, Kong Y, Zhang L, Xie X and Xiao X: Linc01638 promotes tumorigenesis in HER $2^{+}$breast cancer. Curr Cancer Drug Targets 19: 74-80, 2019.

This work is licensed under a Creative Commons Attribution-NonCommercial-NoDerivatives 4.0 International (CC BY-NC-ND 4.0) License. 